\title{
Sir Moses Montefiore (1784-1885), défenseur de la foi
}

Sir Moses Montefiore (1784-1885), Defender of the Faith

Suzy Halimi

\section{(2) OpenEdition}

1 Journals

\section{Édition électronique}

URL : http://journals.openedition.org/rfcb/3638

DOI : $10.4000 /$ rfcb.3638

ISSN : 2429-4373

\section{Éditeur}

CRECIB - Centre de recherche et d'études en civilisation britannique

\section{Édition imprimée}

Date de publication : 1 mars 2013

ISBN : 2-911580-37-0

ISSN : 0248-9015

\section{Référence électronique}

Suzy Halimi, «Sir Moses Montefiore (1784-1885), défenseur de la foi », Revue Française de Civilisation Britannique [En ligne], XVIII-1 | 2013, mis en ligne le 01 mars 2013, consulté le 20 mars 2020. URL http://journals.openedition.org/rfcb/3638 ; DOI : https://doi.org/10.4000/rfcb.3638

Ce document a été généré automatiquement le 20 mars 2020.

\section{cc) (†)}

Revue française de civilisation britannique est mis à disposition selon les termes de la licence Creative Commons Attribution - Pas d'Utilisation Commerciale - Pas de Modification 4.0 International. 


\title{
Sir Moses Montefiore (1784-1885), défenseur de la foi
}

\author{
Sir Moses Montefiore (1784-1885), Defender of the Faith
}

\author{
Suzy Halimi
}

1 Sir Moses Montefiore n'est guère connu des historiens de l'Angleterre victorienne ; son nom n'apparaît pas dans leurs index, jusqu'à la biographie que lui consacre Abigail Green en $2010^{1}$. Ce fut pourtant une éminente personnalité, qui évolua au plus haut niveau de la sphère publique et se tailla une réputation internationale en son temps. Figure emblématique de l'anglo-judaïsme, homme d'une grande piété, philanthrope actif au service des causes humanitaires, notamment aux côtés de Lord Shaftesbury, il était aussi lié d'amitié avec des évangéliques tels que Sir Culling Eardley, avec des quakers, et honoré par des dignitaires de l'Église anglicane, au moment où était votée la loi d'Émancipation des catholiques (1829) et où se développait la campagne pour celle des juifs. Il y aurait là déjà de quoi rappeler l'importance du facteur religieux, à côté des données socio-économiques dans l'histoire d'un pays, ce que s'emploie à démontrer l'historiographie contemporaine.

2 Mais la notoriété de Sir Moses Montefiore ne s'arrêtait pas aux frontières de l'Angleterre. Volant au secours de ses coreligionnaires persécutés de par le monde, il s'était acquis une réputation internationale, de la Russie au Maroc, en passant par Rome, la Syrie et l'Égypte. Prêchant partout la tolérance, précurseur de la défense des droits de l'homme, il pouvait compter sur le soutien de son Gouvernement, sur la sympathie active de l'Empereur des Français, car il en venait à incarner les valeurs morales de l'Occident, messager de la civilisation britannique au rang des grands bâtisseurs de l'Empire. Voyageur infatigable jusqu'à un âge avancé, il faisait en même temps de la Terre Sainte son objectif privilégié, la défense de sa foi restant le fil rouge de toute son action. Ainsi incarne-t-il l'interconnexion entre le local, le national et l'international, lieu générateur des transferts culturels, autre thème de prédilection des historiens d'aujourd'hui.

3 Mais qui est-il au juste, cet être d'exception, et que signifie pour lui l'observance de la Loi mosaïque qui va guider son existence? Répondre à cette question est le prélude 
nécessaire pour comprendre sa croisade de soutien aux opprimés. C'est alors que s'éclaire le rôle qu'il a pu jouer pour la défense de l'orthodoxie dans son propre pays, lors des deux mouvements qui agitent sa communauté au XIXe siècle, mouvements concomitants et interactifs, celui de la réforme religieuse et celui de l'Émancipation politique. Telles seront les trois parties de cette présentation de Sir Moses Montefiore, défenseur de la foi.

\section{L'observance de la Loi}

Sir Moses Montefiore est un homme d'une belle prestance $(1,90 \mathrm{~m})$ et d'une exceptionnelle longévité à l'époque où le «Roi Choléra » fait des ravages à Londres. Jeune, dans son bel uniforme de capitaine, il incarne le gentleman anglais au service de son pays; à l'automne de sa vie, sa calotte noire sur la tête, il fait figure de patriarche biblique. Il est né à Livourne, le 24 octobre 1784, au cours d'un voyage de ses parents en Italie. Il reçoit une éducation privée - il apprend les fondamentaux, lire, écrire et compter, mais aussi le français - et une instruction religieuse sous la houlette de son oncle Mocatta; mais la Bible, la Loi écrite, retient davantage son attention que le Talmud, la Loi orale.

5 Comme les universités et les professions libérales ne sont pas accessibles aux juifs, il travaille d'abord chez un importateur de thé, travail fatigant et peu gratifiant. En 1803, grâce à la générosité d'un membre de sa famille, il devient un des douze juifs autorisés à être courtiers à la Bourse, moyennant l'achat d'une licence auprès des autorités municipales. Ses débuts dans le monde de la finance sont désastreux : d'une honnêteté scrupuleuse, il est victime d'une escroquerie où il perd 30000 livres; il lui faudra près d'une dizaine d'années pour s'en remettre.

6 Mais en 1812, la roue tourne quand il épouse Judith Barent-Cohen, jeune fille accomplie, bien dotée, apparentée à Nathan Mayer Rothschild. Très différents par leur physique et par leur personnalité, les deux hommes se rapprochent pourtant, et Moses devient le courtier en Bourse de son beau-frère. Cette fois, dûment conseillé, investissant dans des opérations fructueuses, il fait fortune ${ }^{2}$ et décide alors de se retirer des affaires pour se consacrer à l'action sociale, devoir religieux à ses yeux, soutenu en cela par son épouse, elle-même d'une grande piété.

7 Entre-temps, en 1809, au moment des guerres napoléoniennes, il s'est engagé dans la milice de son comté, se hissant vite au rang de capitaine. Puis, au fil des années, les responsabilités et les honneurs se succèdent. En 1835, il est élu président du Board of Deputies of British Jews, l'instance qui dirige la communauté juive du royaume, aux côtés du Grand Rabbin, et qui assure le dialogue avec les autorités civiles. Cette fonction, comme chef incontesté de l'anglo-judaïsme, il l'occupe pendant près de quarante ans, jusqu'en 1874. En 1836, il est admis comme fellow de la Royal Society, et l'année suivante, le voilà sheriff de la Cité de Londres, et anobli lorsque la jeune reine Victoria se rend dans la Cité à l'occasion de son couronnement (1837). Une dizaine d'années plus tard, il est fait baronet (1846). Parcours exceptionnel dans la sphère publique, où il fréquente les responsables politiques et le gratin de la société victorienne.

8 Au faîte de la gloire, cet homme qui incarne si bien le capitalisme triomphant de l'Angleterre du XIX ${ }^{e}$ siècle, reste célèbre aussi pour son observance scrupuleuse de sa religion. Certes, dans sa jeunesse, il a trouvé des accommodements avec le Ciel, mais 
lors de son premier voyage en Terre Sainte, en 1827, le sentiment de son identité juive se renforce. À bord du bateau, il adresse une fervente prière à son Créateur :

I humbly pray to the God of my forefathers, my God [...] to grant that I may henceforth become a more righteous and better man, as well as a better Jew [...] This day, I begin a new era. ${ }^{3}$

Dès lors, les exemples de sa piété sont légion. En voyage, il emporte son rouleau de la Torah, son châle de prières, il emmène son cuisinier pour apprêter ses repas dans la stricte observance des lois alimentaires. Le shabbat et les jours de fête, lui et sa femme se rendent à pied à la synagogue, et il en fait de même lors de son investiture comme sheriff de Londres, la cérémonie se déroulant le jour du Nouvel An hébraïque. Voici un extrait de son journal, en 1820, où il détaille le déroulement de ses journées :

With God's blessing, rise, say prayers at 7 o'clock. Breakfast at 9. Attend the Stock Exchange if in London, 10. Dinner 5. Read, write and learn if possible Hebrew and French, 6. Read Bible and say prayers, 10. Then retire. Monday and Thursday mornings attend the synagogue. ${ }^{4}$

10 Mais, par-dessus tout, il observe cette loi suprême du judaïsme, la pratique de la charité. Pour Montefiore, la religion n'est pas simple affaire de prière, de rituel ; elle est action au service de son prochain. Et elle n'est pas un faire-valoir, selon l'analyse du sociologue Marcel Mauss, une façon de désamorcer les antagonismes de classes dans une économie capitaliste. Cette approche fonctionnaliste de la philanthropie n'a aucune pertinence dans le cas de Montefiore. Pour le croyant qu'il est, la charité - la tsedaka, en hébreu - est un acte de justice, un devoir pour les nantis de restituer aux déshérités une partie de la fortune accordée par la Providence.

Riche, Sir Moses est aussi très généreux. Il soulage la misère de ses semblables, sans distinction de religion, en Angleterre comme dans les pays où il se rend. Tel est le plan d'action qu'il se fixe en 1828:

Make a Plan for relieving or affording some Assistance to Poor Persons Endeavour [...] to form some plan for the employment of the Poor [...] Never Refuse to see or hear patiently everyone that applies to me for charity or Assistance and to relieve them as far as prudence will permit. ${ }^{5}$

Il assiste Lord Shaftesbury pour promouvoir l'éducation des enfants pauvres, et le jour de son centième anniversaire, il fait un don pour offrir à ces enfants des ragged schools un jour de fête. En Terre Sainte, où les rescapés des pogroms vivent dans des conditions précaires, il crée routes, écoles, hôpitaux, hospices, avec un souci très victorien de mettre les pauvres au travail et d'éviter l'assistanat: 'I satisfied myself that the inmates were fully deserving of the advantages they were enjoying [...] not neglecting the following of industrial pursuits. ${ }^{\prime 6}$ Il fait construire un hôpital à Nazareth sur le site où Jésus prononça ses sermons. Lorsque l'Angleterre abolit l'esclavage, il est encore là, avec Nathan Rothschild, pour avancer au Gouvernement les fonds nécessaires pour indemniser les propriétaires d'esclaves. Lorsque son épouse décède, en 1862, il rend hommage à celle qui a toujours soutenu son action, et qui en a même été l'instigatrice ${ }^{7}$.

13 Cette générosité sans frontières est saluée unanimement. Quand on organise une cérémonie en son honneur à Ramsgate en 1883, prennent la parole pour faire son éloge le maire et le pasteur, un représentant de la communauté catholique, quatre ministres du culte anglican. Et le 23 octobre 1884, veille de son centième anniversaire, le Times lui consacre son éditorial: 'Englishmen without distinction of creeds contemplate Sir Moses Montefiore's career with as much pleasure as his co-religionists's. Cette notoriété, il la doit aussi à son action à l'étranger, pour la défense de ses coreligionnaires en détresse. 


\section{La Libération des croyants} accusés de meurtre rituel à la suite de la disparition d'un moine italien'. L'affaire fait grand bruit en Europe, et la France, alertée par les articles d'un brillant avocat, Adolphe Crémieux, fait intervenir son consul en Syrie. Aussi, lorsque Sir Moses décide de se rendre sur place, il peut compter sur le soutien de son Gouvernement. Après de nombreuses péripéties liées aux convoitises concurrentes des puissances européennes sur l'Empire ottoman, il est reçu par le sultan et obtient de lui un démenti formel de l'accusation de meurtre rituel, mais aussi un décret promettant la protection de la minorité juive à l'avenir: 'The Jewish nation, dwelling in all parts of our Empire shall be perfectly protected and no person shall molest them [...] in the free exercise of their religion, nor in that which concerns their safety and tranquillity ${ }^{10}$. Copie du décret est remise par Montefiore au cardinal Ravorala, à Rome, à l'empereur Napoléon III et, bien sûr, à la reine Victoria: première démarche, d'envergure européenne, pour la défense des minorités religieuses.

Suivent d'autres voyages, dans le même esprit, et presque toujours avec le même succès. En mars 1846, c'est en Russie, où les juifs, parqués dans les territoires polonais, sont soumis à des exactions par le tsar, qui veut les pousser à la conversion, notamment en enrôlant de force les jeunes gens pendant vingt-cinq ans dans ses armées. Sir Moses, après avoir constaté les faits à Vilnius, fait des dons aux institutions charitables à Varsovie, rencontre le tsar, qui reconnaît avoir été abusé par des conseillers malveillants et s'engage à redresser les torts commis par son administration. En 1858, c'est la traversée des Alpes pour gagner Bologne où l'affaire Mortara fait scandale : un enfant de six ans, Edgardo Mortara, est enlevé à sa famille juive, sous prétexte qu'il aurait été baptisé en secret par la servante catholique. Détresse des parents et de la communauté juive par ailleurs maltraitée dans les États pontificaux, n'ayant accès ni à l'éducation, ni à la charité publique, et vivant sous la menace de baptêmes forcés. Montefiore est appelé à l'aide ; des diplomates anglais et français sont dépêchés auprès du pape Pie IX. Le Saint-Père dit sa compassion pour les parents, mais s'en remet à l'enfant pour une décision, lequel refuse de retourner dans sa famille et sera plus tard missionnaire catholique. C'est un échec pour Montefiore, un échec qui ravive en Angleterre l'hostilité ancestrale au papisme !

bateaux dans le port mettent leurs pavillons en berne. Rongé par le chagrin, menacé de sombrer dans la dépression, Sir Moses reprend sa croisade. En 1863, il se rend au Maroc où la violence récurrente contre les juifs sert d'exutoire aux frustrations des populations locales. Le Daily Telegraph annonce ainsi son départ: 'Old, silverhead as he is, the Jewish Baronet heard of the sufferings of his brethren and took ship for Mogador. Sir Moses was resolved to see the Sultan and ask justice in the name of God and Man' ${ }^{11}$. L'entrevue avec le sultan est un succès : un édit est proclamé, enjoignant à ses sujets de s'abstenir désormais de toute exaction à l'encontre des juifs.

En 1867, Sir Moses est en Roumanie, où sévit une vague d'antisémitisme; en 1872, nouvelle visite en Russie, à la suite d'un pogrom à Odessa. Ici et là, il est accueilli avec les honneurs dus à son âge et à sa renommée, désormais internationale; ici et là, il reçoit des gages d'apaisement quant au sort de ses coreligionnaires. En 1875, c'est le

Revue Française de Civilisation Britannique, XVIII-1 | 2013 
septième et ultime voyage en Terre Sainte, où sévit la famine. Il a 91 ans ! Mais ses plans de développement économique ne recueillent pas, cette fois, l'unanimité : les temps ont changé, et de nouvelles organisations internationales sont entrées en action, animées par des visées plus politiques que par la foi profonde de Sir Moses.

Inutile d'entrer ici, pour notre propos, dans les méandres diplomatiques de ce XIX ${ }^{e}$ siècle, où les grandes puissances rivalisent pour étendre leur zone d'influence dans le monde. L'Angleterre victorienne ne manque pas de grands voyageurs, d'aventuriers qui ont bâti l'Empire. La dimension religieuse de sa vie et de son œuvre fait de Sir Moses Montefiore une figure à part. Lui rêve de construire le royaume de Dieu. Messager de la tolérance, défenseur précoce des droits de l'homme, il incarne les valeurs morales de son pays, ambassadeur de la civilisation britannique auprès de ses interlocuteurs. Le Daily Telegraph insiste sur cet aspect de sa croisade à son retour du Maroc: 'What one good old man had done to wipe away tears from streaming eyes and cause oppression to cease [...] cannot fail to be followed by justice and amity along the shores of Africa ${ }^{12}$. Grâce à lui, les communautés juives en détresse ne subissent plus l'oppression dans l'indifférence générale. Le Gouvernement anglais s'active même en leur faveur. Lorsque Palmerston voit la Russie s'implanter en Terre Sainte pour la défense des orthodoxes et la France pour celle des catholiques, il envisage de placer officiellement les juifs de Palestine sous protection britannique. L'Union Jack, relais de Montefiore au secours du judaïsme! Ironie de l'Histoire, ce zèle se manifeste au moment même où la campagne pour l'Émancipation des juifs se heurte à de nombreuses difficultés sur la scène parlementaire.

\section{La défense de l'orthodoxie}

19 Tandis que Montefiore déploie sa générosité sur le territoire national et à l'étranger, deux problèmes majeurs agitent l'anglo-judaïsme: l'Émancipation, sur le plan politique, et la réforme du rituel sur le plan religieux. Ici et là, le président du Board of Deputies est présent, avec plus de discrétion dans le premier cas, plus de conviction dans le second.

Pendant longtemps, les sujets de Sa Majesté d'obédience non-anglicane ont souffert de restrictions limitant leurs droits civiques, faisant d'eux des citoyens de seconde zone. Peu à peu, ces vexations ont été levées, pour les dissidents d'abord, par l'abrogation des Test and Corporation Acts (1828), puis en 1829, pour les catholiques. Seuls les juifs souffrent encore de discrimination. L'accès aux universités, aux professions libérales leur est interdit, interdite aussi toute participation à la vie politique et aux responsabilités administratives à cause du serment qu'il faut prononcer à la prise de fonction, serment sur les Trente-Neuf Articles avec la formule 'on the true faith of a Christian', deux exigences auxquelles les juifs ne peuvent souscrire, même s'il existe de rares cas où la difficulté a été contournée par artifice ou par tolérance exceptionnelle.

La communauté juive anglaise à l'époque de Montefiore compte 20 à 30000 personnes, pour les deux tiers à Londres. Sa situation socio-économique s'est bien améliorée depuis la Réadmission, au XVII ${ }^{e}$ siècle. Les colporteurs, les fripiers se sont stabilisés dans des commerces plus « honorables ». Une bourgeoisie aisée s'est développée et, au sommet de la pyramide, une élite de familles riches - les Rothschild, les Mocatta, les Montefiore - fréquente sur un pied d'égalité les grands du royaume et même les membres de la famille royale. 

mathématicien Samuel Gompertz, ou David Ricardo, fondateur de l'économie libérale. Les sœurs Adams font vibrer les salles de concert. L'image du juif se modifie de ce fait, surtout depuis la publication, en Allemagne, de Nathan der Weise (1781) de Lessing. On s'éloigne des caricatures du Shylock de Shakespeare, et le Fagin de Dickens prolonge une tradition en voie d'extinction. Des juifs nobles et généreux, on en rencontre sur la scène, dans les romans, sous la plume de Sheridan, de Cumberland, de Maria Edgeworth $^{13}$. Ce philosémitisme se répand chez les évangéliques, même s'il s'assortit, chez eux, du secret espoir de convertir les juifs, prélude à l'avènement des temps messianiques. Ainsi grandit dans l'opinion publique le sentiment qu'il conviendrait de leur accorder désormais la reconnaissance de citoyens de plein droit, d'autant que, de l'autre côté de la Manche, l'abbé Grégoire a brillamment plaidé en ce sens, et en 1807, Napoléon a mis en place avec faste le Sanhédrin. nombreuses propositions de loi, introduites au Parlement, se heurtent à une opposition aux Communes et à la Chambre des Lords, où la résistance est conduite par l'Archevêque de Cantorbéry. Elle est finalement acquise en 1858. Quelle a été la position de Sir Moses Montefiore ? Il est attesté que, dans les années 1830, il a mis son influence au service de la campagne. Mais deux décennies plus tard, il semble plus en retrait; il n'a pas jeté son énergie et sa notoriété dans la balance. C'est qu'il y a en lui une crainte sourde. Gardien de l'orthodoxie, il redoute que l'engagement politique n'entre en conflit avec les exigences de la religion. Ne déclare-t-il pas en 1837, alors même qu'il milite pour l'Émancipation, qu'il est en même temps 'firmly resolved not to give up the smallest part of our religious forms and privileges to obtain civil rights' $?^{14}$ Or, ses appréhensions ne sont pas sans fondements.

La situation religieuse de la communauté juive anglaise n'est pas sans nuages, qui menacent son intégrité et sa cohésion : une dizaine de divorces par an, des conversions au judaïsme de femmes vivant avec des juifs et désireuses de faire légitimer leurs enfants, conversions interdites par le Grand Rabbinat anglais et qu'elles vont chercher sur le continent. Par ailleurs, les conversions de juifs à l'anglicanisme ne sont pas rares. Emblématique est le cas d'Isaac D'Israeli, qui, en conflit avec sa communauté pour une affaire de cotisation, quitte celle-ci et fait baptiser ses enfants, dont Benjamin, futur premier ministre de la reine Victoria. En même temps, il publie The Genius of Judaism (1833), où il fait l'éloge de l'assimilation et critique ceux qui restent attachés à une foi et à des traditions du passé. David Ricardo a, lui aussi, renié la foi de ses ancêtres et épousé une chrétienne ; John King, renonçant à son appartenance religieuse, épouse la comtesse de Lanesborough. Et en même temps, paraissent des ouvrages qui décrivent la vie juive et prônent la conversion au christianisme ${ }^{15}$.

Tout ce ferment se nourrit aussi des nouvelles qui viennent d'Allemagne, où se développe le même courant en faveur d'une réforme du judaïsme traditionnel. Dans les années 1830, les élites juives de Hambourg et de Berlin s'engagent dans une révision non pas tant des dogmes que des pratiques ancestrales : raccourcissement de la liturgie, récitation des prières en allemand et non plus en hébreu, introduction de sermons réguliers dans le service, utilisation des orgues, non-séparation des hommes et des femmes pendant les offices, et à Hambourg, se crée une communauté autonome fondée sur ces pratiques.

Revue Française de Civilisation Britannique, XVIII-1 | 2013 

porte ouverte à la désintégration du peuple juif. Le 10 septembre, les autorités londoniennes, autour du Grand Rabbin, signent un décret d'excommunication à l'encontre des dissidents: 'Any person or persons declaring that he or they reject and do not believe in the authority of the Oral Law, cannot be permitted to have any communion with us, Israelites in any religious rite or sacred $a c t^{17}$. Les avis divergent sur le rôle exact joué par Montefiore dans cette affaire, mais il est sûr que les mesures de rétorsion vont dans le sens de ses convictions, même si, en même temps, il redoute les conséquences néfastes d'une telle décision sur l'avenir de sa communauté. Quand la West London Synagogue demande le droit d'enregistrer ses mariages - droit réservé au Board of Deputies -, c'est un refus catégorique de sa part, et il ajoute : 'I do not consider the place of worship in Burton Street referred to by you to be a synagogue'18. 
Quelques années plus tard, sont élus au Board des représentants de la communauté dissidente - de Manchester. Le président leur refuse le droit de siéger et l'accès à la salle de réunion. Le débat est houleux, et quand la question est soumise au vote des membres, eux-mêmes divisés, c'est la voix du président, prépondérante en l'occurrence, qui tranche en faveur de l'exclusion. Le désordre est alors si indescriptible qu'il faut faire appel à la police pour rétablir le calme. L'unité de l'anglo-judaïsme est bien brisée. L'excommunication ne sera levée qu'en 1874, à la fin du mandat de Sir Moses, et les réformateurs seront admis à siéger au Board après sa mort, en 1886.

\section{Conclusion}

31 Tout au long de sa vie, Moses Montefiore a ainsi été un défenseur convaincu de sa foi. Il l'a pratiquée au quotidien, par une stricte observance de la Loi de Moïse. Il en a honoré l'esprit en cultivant l'amour du prochain et la charité, un des commandements sacrés du judaïsme. Sa foi repose sur le lien indissoluble entre la Loi Écrite - l'Ancien Testament - et la Loi Orale - herméneutique de la première par le Talmud. En ce dixneuvième siècle, qui est, sans conteste, celui de la sécularisation de la vie publique, il témoigne de la permanence du facteur religieux dans la sphère privée, mais aussi dans les corridors du pouvoir. Si les droits de l'homme ont été proclamés par la Révolution française, il a fait de leur défense et illustration sa règle de vie, attribuant toujours ses succès à la divine Providence.

Lorsqu'il s'éteint, le 28 juillet 1885 , les hommages qui lui sont rendus retiennent tous cet aspect de sa personnalité et de son œuvre. Le Times écrit, dans sa notice nécrologique:

It may truly be said that his last thoughts were occupied with the duties of piety, loyalty and benevolence. To the Jews, it may well seem as if, with him, the central pillar of their temple had fallen, but those who calmly contemplate his life will understand that the example of his useful and benevolent career has done its work.

Et dans son oraison funèbre prononcée le 5 août en la synagogue de Bevis Marks, le révérend Hermann Adler conclut sur ces mots : 'The precepts of judaism were the joy of his life.'

Sir Moses Montefiore est peut-être ignoré des historiens de l'Angleterre victorienne ; mais le promeneur qui passe aujourd'hui devant sa maison natale, à Livourne, peut lire cette plaque à sa mémoire: 'On October 241784 was born Sir Moses Montefiore, untiring apostle of tolerance to all kinds of misfortunes, without distinction of race or faith. Died at Ramsgate on July 28. 1885. Honoured by the powerful, blessed by the poor.'

\section{NOTES}

1. Abigail GREEN, Sir Moses Montefiore: Jewish Liberator, Imperial Hero, Cambridge, MA: The Belknap Press of Harvard University Press, 2010. 
2. Il laissera à sa mort une fortune estimée à 374421 livres 2 shillings 5 pence. Voir Geoffrey Alderman, 'Montefiore, Sir Moses Haim (1784-1885), financier and Jewish community leader', in Oxford Dictionary of National Biography, Oxford University Press, 2004: <http:// www.oxforddnb.com.janus.biu.sorbonne.fr/view/article/19042?docPos=4> [consultation le 8 mai 2012].

3. 'Wed. Oct. 24 [1827], At sea', Journal of Sir Moses Montefiore, 1827-1828, Montefiore Family Papers; cité dans Abigail GREEN, 'Rethinking Sir Moses Montefiore: Religion, Nationhood, and International Philanthropy in the Nineteenth Century', The American Historical Review, vol. 110, $\mathrm{n}^{\circ}$ 3, juin 2005, pp. 648-649.

4. Cité dans Ruth SEBAG-MONTEFIORE, A Family Patchwork, London: Weidenfeld \& Nicolson, 1987, p. 109.

5. Arthur Sebag-Montefiore archive, Oxford Centre for Hebrew and Jewish Studies; cité dans GREEN, Sir Moses Montefiore, p. 295.

6. Lettre du 28 août 1866 (London Metropolitan Archives).

7. 'I am no great man. The little good that I have accomplished [...] I am indebted for it to my [...] wife' (The Jewish Chronicle, 19 juin 1863).

8. Cité dans GREEN, 'Rethinking Sir Moses Montefiore', p. 639.

9. Selon une accusation remontant au Moyen Âge, les juifs, lors de la Pâque, commettraient des meurtres pour utiliser le sang de leurs victimes dans la confection du pain azyme.

10. Voir GREEN, Sir Moses Montefiore, p. 150.

11. Repris par The Jewish Chronicle, 19 juin 1863.

12. Cité dans David LITTMAN, 'Mission to Morrocco (1863-1864)', in Sonia LIPMAN \& Vivian D. LIPMAN (eds.), The Century of Moses Montefiore, Oxford: Oxford University Press, 1985, p. 192.

13. A titre d'exemples, citons The Jew of Mogador (1808) de Richard Cumberland, Ivanhoe (1819) de Walter Scott, l'amitié entre Isaac Nathan et Lord Byron, qui écrivit 29 poèmes pour servir de texte à des mélodies juives, Leigh Hunt, grand admirateur de la langue et de la littérature juives, etc.

14. Louis Loewe (ed.), Diaries of Sir Moses and Lady Montefiore [1890], 2 vols., London: Jewish Historical Society of England, 1983, vol. 1, p. 111.

15. Voir David KATZ, The Jews in the History of England, 1485-1850, Oxford: Clarendon Press, 1994, p. 351.

16. Dr. Nathan Marcus ADLER, Solomon's Judgment: A Sermon Delivered at the Great Synagogue on the Sabbath of Dedication, 31. December, 1853, London, 1854, p.12; cité dans Bill WILLIAMS, The Making of Manchester Jewry, 1740-1875 [1976], Manchester: Manchester University Press, 1985, p. 225.

17. Pour le texte complet de cette proclamation, voir Arthur BARNETT, The Western Synagogue through two Centuries (1761-1961), London: Valentine Mitchell, 1961, p. 180.

18. Lettre à Francis Goldsmid (8 février 1842), in GREEN, Sir Moses Montefiore, p. 166.

\section{RÉSUMÉS}

Sir Moses Montefiore est peu connu des historiens de l'Angleterre victorienne. C'était pourtant une personnalité importante de l'époque, introduit et honoré dans les cercles politiques, sociaux et religieux de son temps. Juif orthodoxe, il observait scrupuleusement les commandements de sa foi dans sa vie quotidienne, en particulier une des lois fondamentales du judaïsme, la pratique de 
la charité. Possédant une importante fortune, il la mettait au service des déshérités, sans distinction de religion, soutenant par exemple le grand philanthrope Lord Shaftesbury pour le développement de l'éducation des enfants pauvres. Son action s'étendait aussi par-delà les frontières, lorsqu'il volait au secours de ses coreligionnaires persécutés de par le monde. Soutenu dans cette action par le Gouvernement de son pays, il devint le porte-parole de la tolérance, de la défense des droits de l'homme, des valeurs de la civilisation britannique.

Lorsque la question de l'Émancipation des juifs fut âprement discutée au Parlement de Westminster, il soutint le mouvement en sa qualité de président du Jewish Board of Deputies, mais non sans craindre que la conquête des droits civiques ne vienne empiéter sur une stricte observance de la foi. Et lorsque l'orthodoxie traditionnelle fut remise en question par ceux qui voulaient «moderniser » le rituel du judaïsme dans l'espoir de promouvoir l'Émancipation, Sir Moses Montefiore s'opposa violemment à ces «hérétiques", refusant de les admettre comme membres du Board aussi longtemps qu'il en demeura le président.

Sir Moses Montefiore is hardly to be found in the books of the historians of Victorian Britain. He was however an important figure of the time, a friend of many important personalities in the political, social and religious circles. An orthodox Jew, he strictly observed the dictates of his faith in his daily life and especially that basic law of Judaism, the practice of charity. A very rich man, he used his money for the relief of the poor, without any distinction of religion, supporting, for instance, the great philanthropist, Lord Shaftesbury, in the development of education for destitute children. His action also reached beyond the seas, when he flew to the rescue of his coreligionists persecuted in several parts of the world. Supported by his Government, he thus became the mouthpiece of tolerance, of the rights of man and of the values of British civilization. When the question of Jewish Emancipation was hotly discussed in Parliament, he supported the movement as President of the British Board of Deputies, fearing at the same time that the conquest of civil rights might encroach upon a strict observance of his faith. And when the traditional orthodoxy was questioned by a number of Jews, eager to 'modernize' the practice of Judaism in order to promote Emancipation, then he was deadly against those 'heretics' and strongly opposed their admission to the Board as long as he remained its President.

\section{AUTEUR}

\section{SUZY HALIMI}

Université Sorbonne Nouvelle - Paris 3 